\title{
Clinical application of AIMS65 score to predict outcome in patients with acute upper gastrointestinal bleeding
}

\author{
Kijan Maharjan', Rajesh Kumar Mandal', Sanjay Shrestha' \\ 'Department Of Internal Medicine, Sukraraj Tropical and Infectious Disease Hospital,Teku, Nepal \\ ${ }^{2}$ Department of Internal Medicine, Bheri Hospital, Nepalgunj, Nepal
}

\begin{abstract}
This work is licensed under a Creative Commons Attribution 4.0 Unported License.

\section{BACKGROUND}

Acute upper gastrointestinal bleeding (AUGIB) is a potentially life-threatening condition that requires rapid assessment in the emergency department. The current available scores are complex and have not been widely used in clinical practice. AIMS65 score is a simple score that can be used to risk stratify patients with AUGIB.
\end{abstract}

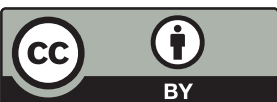

\section{METHODS}

This was a descriptive cross-sectional study done at a single tertiary centre, NAMS, Bir Hospital among the patients presenting with AUGIB from August 2018 to January 2019. AIMS65 scores were calculated in patients presenting with acute UGIB by allotting 1 point each for albumin level $<3 \mathrm{~g} / \mathrm{dl}$, INR $>1.5$, alteration in mental status, systolic blood pressure $\leq 90 \mathrm{~mm}$ $\mathrm{Hg}$, and age $\geq 65$ years. Risk stratification was done during the initial 12 hours of hospital admission.

\section{RESULTS}

A total of 84 patients consisting of 68 males and 16 females were enrolled in our study, with age ranging from 27 to 80 years. ICU admission, endoscopic therapy and blood transfusion were required in 22,44 and 49 patients respectively. In-patient mortality, the need for blood transfusion, endoscopic therapy or ICU admission were higher in those with AIMS65 score $\geq 2$ showing statistically significant positive association $(p=0.000)$.

\section{CONCLUSION}

AIMS65 score is a simple non-endoscopic risk score that can be applied in patients of acute upper gastrointestinal bleeding to risk stratify and to predict in-patient mortality, the need for blood transfusion, endoscopic therapy or ICU admission.

\section{KEYWORDS}

AIMS65 score; Upper gastrointestinal bleeding; Endoscopy; Mortality

\section{INTRODUCTION}

Upper gastrointestinal bleeding (UGIB) is a gastrointestinal emergency that can result in significant morbidity, mortality, and use of health care resources. The etiology of UGIB can vary from trivial causes like gastric erosions to potentially fatal conditions like aorto-enteric fistula. ${ }^{?}$

Identification of high risk patients can help to predict those requiring endoscopic intervention or intensive care. For this, many risk scoring systems like Glasgow-Blatchford score (GBS), Rockall risk score (RS), and AIMS65 score (AIMS65) have been developed among which AIMS65 is relatively simple and easy to use. ${ }^{2}$
The AIMS65 score consists of five factors: serum albumin , INR, altered mental status, systolic blood pressure and age where each factor is assigned a score of $1 .{ }^{3}$ AIMS65 score $>1$ : high risk patients, AIMS65 score $>2$ : consider ICU management and an urgent endoscopy following adequate volume resuscitation, while AIMS65 score of 0 can be considered for outpatient management. ${ }^{4}$

AIMS65 is comparable to GBS and RS in essential endpoints

\section{*Corresponding Author}

Dr. Kijan Maharjan,Department of Internal Medicine, Sukraraj Tropical and Infectious Hospital, Teku, Kathmandu, Nepal, Email: kijan1069@gmail.com Phone: +977-9841560494 
such as inpatient mortality, the need for endoscopic intervention and the selection of high risk patients. ${ }^{5}$ In the current study, we aim to evaluate the prognostic value of AIMS65 score in patients with UGIB.

\section{METHODS}

This was a descriptive cross-sectional hospital based study conducted at department of Gastroenterology and Hepatology at National Academy of Medical Sciences (NAMS) , Bir hospital, Mahabouddha, Kathmandu from August 2018 to January 2019. The ethical Clearance was taken from the Institutional Review Board (IRB), NAMS, Bir Hospital. A written consent was taken from all the patients before enrollment in the study.

\section{Inclusion criteria:}

Patients above 14 years of age.

Patients with acute UGIB presenting within 24 hours of hemorrhage onset.

Patients who were admitted in department of Hepatology and Gastroenterology at Bir hospital.

Endoscopic evaluation done after hospital admission.

\section{Exclusion criteria:}

Patients denying for consent.

Patients with late presentation (>24 hours) after UGIB onset.

Sample size :

The sample size was calculated using the following formula $n=z 2 p(1-p) / d 2$

Where, $\mathrm{n}=$ required sample size,

$z=$ statistical value for a level of confidence (for 95\% level of confidence, $z=1.96$ )

$p=$ area under the receiver operating characteristic curve

$\mathrm{d}=$ precision or maximum tolerable error

Area under the receiver operating characteristic curve for AIMS65 score to predict intervention or death in UGI bleeding in one study was $0.68 .{ }^{6}$ Hence considering $z=1.96$, $p=0.68$ and $d=0.1$ (precision of $10 \%$ ), total sample size would be around 84 .

Consecutive sampling technique was applied. Patients diagnosed with UGIB who were admitted in NAMS, Bir hospital were included in the study after obtaining informed consent. Data were collected using a structured Proforma covering the relevant details. A detail history was taken and patients underwent a clinical examination, laboratory evaluation and UGI endoscopy which were performed by a trained experienced personnel of department of gastroenterology and hepatology. As per the protocol If any endoscopic intervention were needed, they were accomplished during endoscopic procedure such as variceal ligation. Necessary interventions (blood transfusion, endoscopic intervention) during the course of management, ICU admission or mortality were noted. Data were entered in the tabulated format.

Statistical Analysis:

SPSS version 20 was used for data analysis. Statistical significance of the result were assessed. For the purpose of this study, 95\% confidence interval was accepted and $p$ value of $<0.05$ was taken as significant. Descriptive statistics (frequency, percentage) were used and Chi-square test was used to assess correlation between AIMS65 score and outcome in the form of in-hospital mortality, blood transfusion, ICU admission or Endoscopic therapy.

\section{RESULTS}

A total of 84 patients were enrolled in this study. Out of which,68(81\%) were male and 16 (19\%) were female. Among the patients presented with acute upper gastrointestinal bleeding, the number of male were higher than that of female. $61.9 \%$ of patients were in $40-59$ age group followed by $26.1 \%$ above 60 of age. The most common presenting complain was malena $(84.5 \%)$ followed by anemia, hemetemesis and shock, $79.7 \%, 52.4 \%$ and $41.6 \%$ respectively. On endoscopy the commonest cause of bleeding was found to be gastroesophageal varices $52.3 \%$ followed by peptic ulcer $20.2 \%$ and erosive gastritis $16.6 \%$ respectively. The patient characteristics are given in Table 1.

Table 1: Characteristics of Patients of Upper gastrointestinal Bleeding.

\begin{tabular}{|l|l|l|l|}
\hline Variable & \multicolumn{1}{|c|}{ Characteristics } & Frequency & \multicolumn{1}{c|}{$\%$} \\
\hline Sex & Male & 68 & 81 \\
& Female & 16 & 19 \\
\hline Age Group & $21-39$ & 10 & 11.9 \\
& $40-59$ & 52 & 61.9 \\
& $>60$ & 22 & 26.1 \\
\hline Presenting & Hemetemesis & 44 & 52.4 \\
Complain & Malena & 71 & 84.5 \\
& Anemia & 67 & 79.7 \\
& Shock & 35 & 41.6 \\
\hline Etiology of & Peptic ulcer & 17 & 20.2 \\
UGl Bleed & Gastroesophageal varices & 44 & 52.3 \\
& Erosive gastritis & 14 & 16.6 \\
& Mallory weiss tear & 2 & 2.3 \\
& Gastric carcinoma & 5 & 5.9 \\
& Portal Hypertensive & 2 & 2.3 \\
& Gastropathy & & \\
\hline
\end{tabular}


The Patients were admitted in the ward and icu according to their severity and was resuscitated and underwent blood transfusion and endoscopic interventions. 17 out of 84 patients expired during the course of treatment. The distribution of patients with respect to outcomes are given in Table 2.

Table 2: Distribution of the study population with Outcome variables based on AIMS65 Score 0-5

\begin{tabular}{|c|c|c|c|c|c|c|c|}
\hline \multirow[t]{2}{*}{ Outcome Variable } & \multicolumn{6}{|c|}{ AIMS65 Score $0-5$} & \multirow{2}{*}{ Total } \\
\hline & 0 & 1 & 2 & 3 & 4 & 5 & \\
\hline Blood Transfusion & 2 & 3 & 16 & 11 & 13 & 4 & 49 \\
\hline Endoscopic Therapy & 0 & 0 & 16 & 11 & 13 & 4 & 44 \\
\hline ICU admission & 0 & 0 & 2 & 3 & 13 & 4 & 22 \\
\hline In-patient Mortality & 0 & 0 & 0 & 2 & 11 & 4 & 17 \\
\hline Total & 19 & 13 & 24 & 11 & 13 & 4 & 84 \\
\hline
\end{tabular}

49 out of total 84 cases got blood transfusion. Only 2 out of 19 and 3 out of 13 with AIMS65 score 0 and 1 got blood transfusion. However 16 out of 24 with score 2 got transfusion. Each patient with AIMS65 score 3,4 and 5 got blood transfusion.

It was seen that none case with AIMS65 score 0 or 1 required endoscopic therapy. All cases with score 3, 4 and 5 underwent endoscopic intervention while 16 out of 24 cases with score of 2 had undergone endoscopy.

The study showed that no patients with AIMS65 score of 0 and 1 required ICU care. 2 out of 24 with score of 2 and 3 out of 11 with score of $3 \mathrm{got}$ ICU admission. All of the cases; 13 and 4 with score of 4 and 5 respectively required ICU care.

The study revealed there was no mortality in patients with AIMS65 score of 0,1 and 2. However 2 out of 11, 11 out of 13 and 4 out of 4 mortality cases among patients with score of 3, 4 and 5 respectively. The mortality rate was found to be 0 in patients with score of 0,1 and 2; and 22.2\%, 84.6\% and $100 \%$ in patients with score of 3,4 and 5 respectively.

AIMS65 score less than 2 and $\geq 2$ has relationship with the outcome variables. The distribution is given in the Table 3 .

Table 3: Distribution of clinical outcomes based on AIMS65 Score $<2$ and $\geq 2$

\begin{tabular}{|l|l|l|l|l|l|}
\hline \multicolumn{1}{|c|}{ AlMS65 Score } & $\begin{array}{c}\text { Blood Transfusion } \\
\text { Received }\end{array}$ & Endoscopic Therapy & \multicolumn{1}{|c|}{ ICU Admission } & In -patient mortality & Total \\
\hline$<2$ & $5(15.6 \%)$ & 0 & 0 & 0 & 32 \\
\hline$\geq 2$ & $44(84.6 \%)$ & $44(84.6 \%)$ & $22(42.3 \%)$ & $17(32.7 \%)$ & 52 \\
\hline Total & 49 & 44 & 22 & & 84 \\
\hline
\end{tabular}

The table shows $5(15.6 \%)$ out of 32 patients with AIMS65 score of less than 2 received blood transfusion while 27 (84.4\%) patients did not. Among 52 cases with score $\geq 2,44$ (84.6\%) got transfused and 8 (15.4\%) cases did not. This indicates that patients with AIMS65 score $\geq 2$ has higher probability for need of blood transfusion and vice-versa.

Similarly, the cases with score less than 2 did not require Endoscopic intervention while among 52 cases with score 2 or more, 44 (84.6\%) required Endoscopic therapy and $8(15.4 \%)$ cases did not. This indicates that patients with AIMS65 score $\geq 2$ had higher probability for need of endoscopic therapy and vice-versa.

The data showed that no patients with AIMS65 score of less than 2 required ICU care while 22 (42.3\%) out of 52 patients with score of 2 or more required ICU admission. This data indicates that patients with AIMS65 score of less than 2 probably does not need ICU care.

The table shows that there was no mortality among cases with AIMS65 score of less than 2 while 17 (32.7\%) out of 52 cases with score of 2 or more had in-patient mortality. This signifies that the patients with upper gastrointestinal bleeding with AIMS65 score of less than 2 will not probably lead to in-patient mortality.

Table 4: AIMS65 Score in relation to different clinical outcome in patients with UGIB

\begin{tabular}{|l|l|l|l|}
\hline \multicolumn{1}{|c|}{$\begin{array}{c}\text { AIMS65 } \\
\text { Score }\end{array}$} & \multicolumn{2}{|c|}{$\begin{array}{c}\text { Outcome (blood transfusion, Endoscopic } \\
\text { therapy, ICU admission, In-patient mortality) }\end{array}$} & Total \\
\hline & \multicolumn{1}{|c|}{ Yes } & \multicolumn{1}{|c|}{ No } & \\
\hline$<2$ & $5(15.6 \%)$ & $27(84.4 \%)$ & 32 \\
\hline$\geq 2$ & $50(96.2 \%)$ & $2(3.8 \%)$ & 52 \\
\hline Total & 55 & 29 & 84 \\
\hline
\end{tabular}

Chi-Square value is 56.830, df is 1 and $p$-value is 0.000

The data showed that 5 (15.6\%) out of 32 patients who had AIMS65 score less than 2 required blood transfusion, undergone endoscopic therapy, required ICU care or had inpatient mortality. And 50 (96.2\%) out of 52 cases with score of 2 or more required above mentioned intervention or had in-patient mortality. This data signifies that those patients presenting with acute upper gastrointestinal bleeding with AIMS65 score 2 or more at presentation likely require blood transfusion, endoscopic therapy or ICU care or will have mortality and vice versa.

\section{DISCUSSION}

In our study in 84 patients presenting with acute gastrointestinal bleeding in Bir Hospital, we found that the AIMS65 is a simple, non-endoscopic risk score that can be 
applied in patients with acute UGIB. In this study, the score predicted high in-hospital mortality, blood transfusion, ICU admission, and endoscopic therapy in patients with AIMS65 score of 2 or more.

Our study included 84 patients out of which 32 had AIMS65 score of less than 2 and 52 had score of 2 or more. The data showed that among 32 patients who had AIMS65 score of less than 2 27(84.4\%) did not require blood transfusion, all 32 (100\%) did not require endoscopic therapy and ICU care and none of those 32(100\%) had in-patient mortality.

Among 52 patients who had AIMS65 score of 2 or more, $44(84.6 \%)$ received blood transfusion and also same number of patients 44(84.6\%) required endoscopic therapy, 22(42.3\%) needed ICU care and 17(32.7\%) had in-patient mortality.

Saltzman et al. $^{7}$ reported that the patients with no risk factors had a low mortality rate (0.3\%) and those with all five risk factors had a high mortality rate $(24.5 \%)$. Our results showed 0 mortality in patients with no risk factors and $100 \%$ mortality in those with all five risk factors. Hence, our results generally agreed with the findings of Saltzman et al. However, our study group had lower proportion of patients with non-variceal bleeding (47.7\%) compared to the study by Saltzman et al (98.3\%).

In other study done in patients with non-variceal upper GI bleeding, mortality rates increased with higher AIMS65 scores. No deaths occurred among patients with AIMS65 scores of 0 and 1. For patients with AIMS65 scores of 2, 3,4 , and 5 , mortality rates were $0.9 \%, 1.5 \%, 9.5 \%$, and $50.0 \%$, respectively. ${ }^{8}$ Our study agrees with this study as the mortality rate was found to be 0 in patients with score of 0,1 and 2 ; and $22.2 \%, 84.6 \%$ and $100 \%$ in patients with score of 3, 4 and 5 respectively in our study. However, our study includes study population with both variceal and nonvariceal UGI bleeding.

In one study done in 251 patients, patient groups with AIMS65 scores <2 and $\geq 2$, blood transfusion (69.4\% vs. $87.3 \%, p=0.008)$, ICU stay (16.8\% vs. $38.2 \%, p=0.001)$, and mortality were significantly higher in the latter group (4.5\% vs $30.9 \%)$. The mortality rate in patients with AIMS65 scores of $0,1,2,3$, and 4 were $3 \%, 7.8 \%, 20 \%, 36 \%$, and $40 \%$, respectively. ${ }^{\prime}$ Our study agrees with this study as our study results showed in patient group with AIMS65 score $<2$ and $\geq 2$, blood transfusion(15.6\% vs 84.6\%),endoscopic therapy (0 vs $84.6 \%)$, ICU stay(0 vs $42.3 \%)$ ) and mortality rate in patients with AIMS65 scores of $0,1,2,3$, and 4 were $0 \%$, $0 \%, 0 \%, 22.2 \%$, and $84.6 \%$, respectively; significantly higher in the latter group(0 vs 32.7\%).

However, some studies showed that AIMS65 score was not suitable for predicting outcome in patient with UGI bleeding.
In a study by Jung et al. ${ }^{9}$ that included only patients with bleeding peptic ulcers; the AIMS65 score showed lower predictive accuracy for clinical outcomes. Their single center retrospective study ( $n=149$ ) of patients with bleeding peptic ulcers found that the AIMS65 score was not suitable for predicting outcomes in this patient population (area under the curve, $0.571 ; 95 \% \mathrm{Cl}, 0.49$ to 0.65$)$. They found low serum albumin levels to be a risk factor associated with high mortality in these patients. They explained the discordance in their results by the diverse etiology (mixed patient population with both variceal and non-variceal UGIB) for GI hemorrhage in the study by Saltzman et al. ${ }^{7}$

Many studies have been performed worldwide for applicability of AIMS65 score comparing with other scores like GBS to predict outcome in UGI bleeding though we had not done comparison in our study. In a comparative study of AIMS65 score and GBS, Hyett et al. ${ }^{10}(n=278)$ found that the AIMS65 score was superior in predicting inpatient mortality from UGIB, while the GBS was superior for predicting the need for blood transfusion. Both scores were similar in predicting the composite clinical endpoint (inpatient mortality; rebleeding and endoscopic, radiologic, or surgical intervention), ICU admission, rebleeding, length of stay, and timing of endoscopy.

A retrospective study in Japan looked at 192 patients who presented with GI bleeding and found high AIMS65 scores to be a good predictor of prognosis in comparison with the GBS. ${ }^{11}$ But, this was a relatively small study that furthermore included patients presenting with lower GI bleeding, a condition for which neither the GBS nor AIMS65 score is validated.

Whether the AIMS65 score is applicable for predicting outcomes in patients of non-variceal GI bleeding remains uncertain, since 2 of the 5 risk factors in AIMS65 scores are generally accepted as poor prognostic factors of liver cirrhosis, i.e. serum albumin $<3.0 \mathrm{~g} / \mathrm{dL}$ and $\mathrm{INR}>1.5 .{ }^{9}$ But two reports, Saltzman et al. ${ }^{7}$ and Hyett et al. ${ }^{10}$ confirmed the applicability of AIMS65 in acute upper GI bleeding patients, including bleeding of variceal and non-variceal origin. And our study also agrees on it.

Except the study by Jung et al, ${ }^{9}$ most of the studies including ours showed that AIMS65 score can be applied in patients with acute upper gastrointestinal bleeding to predict in-hospital mortality, the need for blood transfusion, ICU admission and endoscopic therapy.

\section{CONCLUSION}

In conclusion, the AIMS65 is a simple non-endoscopic risk score that can be applied to patients with acute upper gastrointestinal bleeding. This score can be used successfully after hospital admission, thus assisting in 
early decision-making and triage. AIMS65 score $\geq 2$ predicts high in-hospital mortality, blood transfusion, ICU admission or endoscopic therapy.

\section{Conflict of Interest: None}

\section{ACKNOWLEDGEMENTS: None}

\section{REFERENCES}

1. Thandassery RB, Sharma M, John AK, Al-ejji KM, Wani H, Sultan K. Clinical Application of AIMS65 Scores to Predict Outcomes in Patients with Upper Gastrointestinal Hemorrhage. Clin Endosc.2015;48:380-384.

2. Cho HS, Han DS, Ahn SB, Byun TJ, Kim TY, Eun CS, et al. Comparison of the effectiveness of interventional endoscopy in bleeding peptic ulcer disease according to the timing of endoscopy. Gut Liver. 2009;3(4):266-70.

3. Venkat A, Cattamanchi S, Madali A, Farook AR, V. Trichur R. Comparison of the AIMS-65 Score with the Glasgow-Blatchford Score in Upper Gastrointestinal Bleed in the Emergency Department. Eurasian J Emerg Med [Internet]. 2017;16(2):708.

4. Rotman S.R., Saltzman J.R. (2017) Nonvariceal Upper Gastrointestinal Bleeding. In: Adler D. (eds) Upper Endoscopy for GI Fellows. Springer, Cham.

5. Juan G Martı́nez-Cara, Rita Jime'nez-Rosales, Margarita U' beda-Mun Oz, Comparison of AIMS65, Glasgow-Blatchford score, and Rockall score in a European series of patients with upper gastrointestinal bleeding: performance when predicting in-hospital and delayed mortality United European Gastroenterology Journal0(0) 1-9.

6. Adrian J Stanley, Loren Laine, Harry R Dalton, Jing H Ngu, Michael Schultz, Roseta Abazi, et al. Comparison of risk scoring systems for patients presenting with upper gastrointestinal bleeding. BMJ 2017;356:i6432.

7. Saltzman JR, Tabak YP, Hyett BH, Sun X, Travis AC, Johannes RS. A simple risk score accurately predicts in-hospital mortality, length of stay, and cost in acute upper GI bleeding. Gastrointest Endosc 2011;74:1215- 1224

8. Park SW, Song YW, Tak DH, Ahn BM, Kang SH, Moon HS, et al.The AIMS65 Score Is a Useful Predictor of Mortality in Patients with Nonvariceal Upper Gastrointestinal Bleeding: Urgent Endoscopy in Patients with High AIMS65 Scores.Clin Endosc.2015;48:522-527.

9. Jung $\mathrm{SH}, \mathrm{Oh} \mathrm{JH}$, Lee HY. Is the AIMS65 score useful in predicting outcomes in peptic ulcer bleeding? World J Gastroenterol 2014;20:1846- 1851 .

10. Hyett BH, Abougergi MS, Charpentier JP. The AIMS65 score compared with the Glasgow-Blatchford score in predicting outcomes in upper GI bleeding. Gastrointest Endosc 2013;77:551-557
11. Nakamura S, Matsumoto T, Sugimori H, Esaki M, Kitazono $T$, Hashi-zume M. Emergency endoscopy for acute gastrointestinal bleeding: prognostic value of endoscopic hemostasis and the AIMS65 score in Japanese patients. Dig Endosc 2014;26:369-376. 